\title{
Mindfulness Training Disrupts Pavlovian Conditioning
}

2

3

Adam Hanley ${ }^{1,2}$,

Eric Garland ${ }^{1,2 *}$.

5

${ }^{1}$ Center on Mindfulness and Integrative Health Intervention Development (C-MIIND),

7 University of Utah.

8

${ }^{2}$ College of Social Work, University of Utah.

$9 \quad *$ Correspondence to: eric.garland@socwk.utah.edu. 


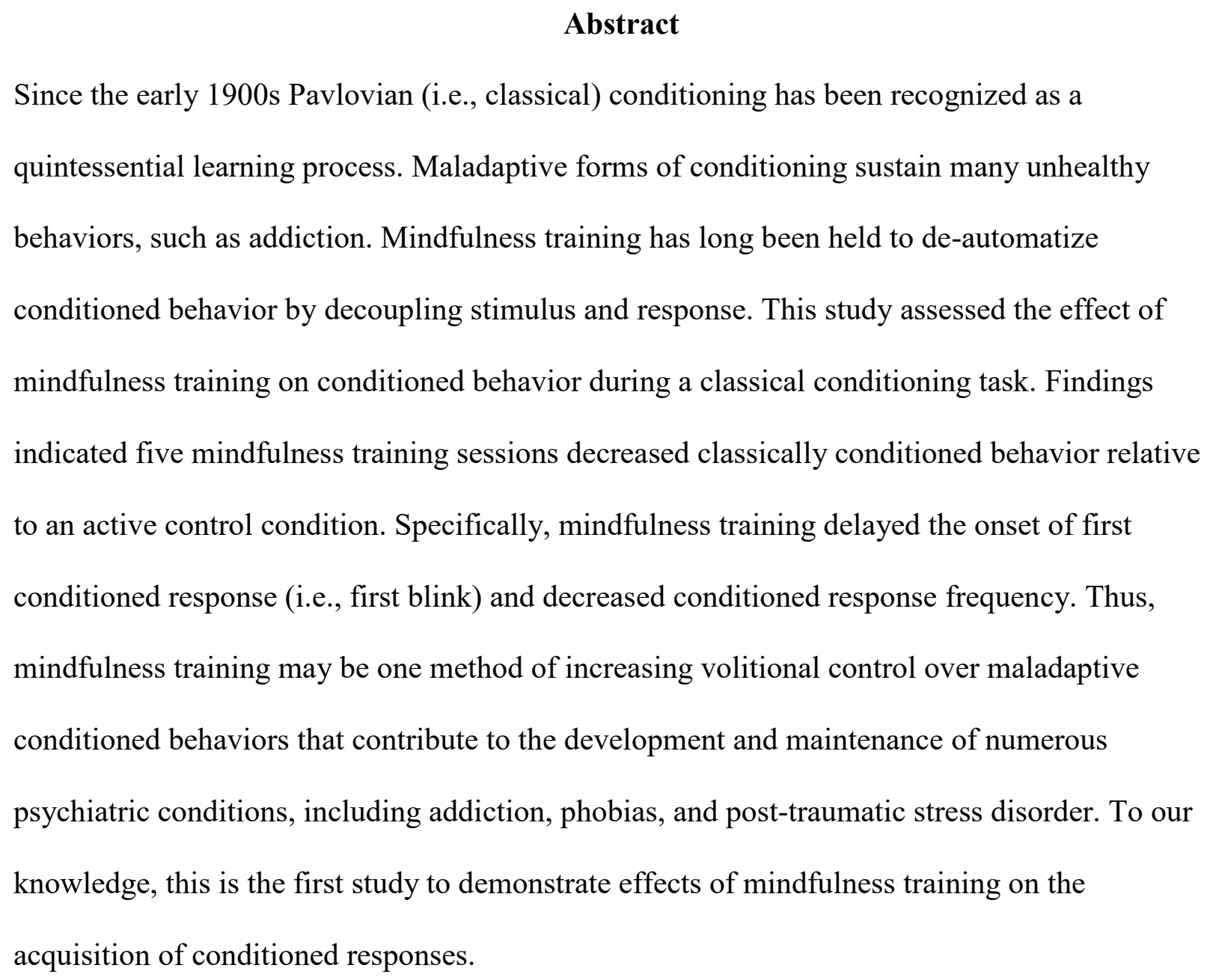

Keywords: Classical Conditioning, Mindfulness, Meditation, Behavioral Therapy 


\section{Introduction}

In the early 1900s, Ivan Pavlov delineated the quintessential learning process of classical conditioning (Pavlov, 2010). Classical conditioning involves pairing two stimuli, an unconditioned stimulus (e.g., appetizing food) that naturally elicits a response (e.g., salivation) with a conditioned stimulus (e.g., bell), to create a new, learned response (e.g., salivation when hearing the bell). Many adaptive behaviors are learned through conditioning; however, maladaptive forms of conditioning are also common. Maladaptive conditioning contributes to the development and maintenance of "bad habits" and psychiatric conditions, including addiction (Hogarth, Balleine, Corbit, \& Killcross, 2013; Wikler, 1948), phobias (Davey, 1992; Watson \& Rayner, 1920), and post-traumatic stress disorder (Pitman, 1989; VanElzakker, Dahlgren, Davis, Dubois, \& Shin, 2014). Since Pavlov, extensive research has examined the neuroanatomical regions implicated in conditioning (Berger, Alger, \& Thompson, 1976; Cheng, Disterhoft, Power, Ellis, \& Desmond, 2008), and translated conditioning processes (Craske et al., 2008; Maren, 2011) into efficacious behavioral therapies designed to extinguish maladaptive conditioned behaviors (Hofmann \& Smits, 2008; Powers, Halpern, Ferenschak, Gillihan, \& Foa, 2010). However, no behavioral therapy has been found to disrupt the acquisition of classically conditioned responses. The current study provides novel empirical evidence that mindfulness meditation training, a common behavioral therapy technique, disrupts learning of classically conditioned behavior.

Mindfulness is a mental state in which one non-reactively attends to present-moment experience with an attitude of acceptance (Kabat-Zinn, 1990). Traditionally construed as a means of de-automatizing conditioned behavior (Deikman, 1966), mindfulness is theorized to decouple stimulus and response (Shapiro, Carlson, Astin, \& Freedman, 2006). Mindfulness may 
ameliorate unhealthy cognitive and behavioral habits (Garland, Farb, R. Goldin, \& Fredrickson, 2015) by increasing sensitivity to currently operative contingencies and relaxing the control of previously established contingencies (McHugh, Procter, Herzog, Schock, \& Reed, 2012). In this manner, mindfulness meditation de-automatizes behavior by cultivating a "fresh awareness" (Hanh, 2009) of present moment experience unconstrained by old, conditioned habits. Evidence indicates that a propensity toward mindfulness in everyday life impedes conditioned learning of complex, probabilistic visual (Stillman, Feldman, Wambach, Howard, \& Howard, 2014) and grammatical (Whitmarsh, Uddén, Barendregt, \& Petersson, 2013) patterns, that long-term mindfulness meditators more quickly relinquish conditioned reward expectations associated with learned cues (Kirk \& Montague, 2015), and that a mindfulness-based treatment for addictive behaviors reduces attentional reactivity to conditioned drug cues (Garland, Baker, \& Howard, 2017). Yet, to date no study has investigated the effect of mindfulness meditation training on the acquisition of conditioned behavioral responses.

This study assessed the effect of mindfulness training on conditioned behavior during a canonical classical conditioning task, delay eyeblink conditioning, in a sample of healthy, young adults. This methodology allowed for investigation of whether mindfulness disrupts classical conditioning through the process of de-automatization (Deikman, 1966). Conditioned learning rates, quantified by conditioned blink frequency during the delay eyeblink conditioning task, was the primary outcome of interest. We hypothesized that relative to an attention control condition, mindfulness training would be associated with significantly lower conditioned blink frequency. Secondarily, we hypothesized that mindfulness training would be associated with significantly longer time to acquire the first conditioned response. Finally, we hypothesized that the effect of mindfulness training on conditioned blink responses would be mediated by increases in state 
mindfulness and independent of the effects of mindfulness on parasympathetic regulation of startle responses induced by the task.

\section{Method}

A sample size of 48 was determined to provide sufficient power $(1-\beta=0.90)$ to observe a small effect size $\left(\eta^{2}=.04\right)$ in conditioned response frequency. Oversampling by $10 \%$ to account for potential screen failures, we recruited 53 participants for an attention training study at a large university in the Western United States. Recruitment materials did not mention mindfulness. Four participants were excluded from the study following screening due to hearing impairment that would have prevented acquisition of conditioned responses by auditory stimuli. The final data set consisted of 49 healthy participants (36 females) between 18 and 43 years of age $(\bar{x}=27.17, S D=6.46)$ without memory disorders or learning disability diagnoses. The local Institutional Review Board approved all study procedures and all participants provided written informed consent.

Study procedures took place over the course of six, individually scheduled study sessions, completed over three weeks. In the first session, consent and demographic information were obtained before participants completed a five-minute resting baseline assessment in which they remained still, with their eyes open and did not speak. Following this rest period, participants completed a self-report measure of state mindfulness (Lau et al., 2006). The next four study sessions lasted 15 minutes each and included an 11-minute experimental induction - either mindfulness training or active listening. Immediately following the induction, the same state mindfulness measure completed during the first session was used as a manipulation check to characterize the extent of state mindfulness produced by the induction. Participants randomized to the mindfulness condition $(n=24)$ received scripted instruction in basic mindful breathing and 
body scanning techniques (Kabat-Zinn, 1990). Participants randomized to the active listening control condition $(\mathrm{n}=25)$ listened to time-matched selections from The Natural History of Selborne (White, 2004) - an attention control condition validated by Zeidan et al. (Zeidan, Johnson, Diamond, David, \& Goolkasian, 2010) for use in experimental studies of mindfulness inductions. In the sixth session, the delay eyeblink conditioning task was performed after participants completed their respective experimental induction and self-report measures. Electrocardiogram (ECG) sensors were affixed to participants' pectoral muscles and ECG was recorded during experimental induction and the eyeblink conditioning task.

An empirically-validated delay eyeblink conditioning procedure was used (Daum et al., 1993; Wolf, Minnebusch, \& Daum, 2009). Participants completed six conditioning blocks followed by one extinction block. Each block consisted of 10 trials. The conditioning blocks were comprised of seven reinforced and three unreinforced trials ( $70 \%$ reinforcement schedule), presented in random order (Figure 1a). The extinction block consisted only of unreinforced trials. For each reinforced trail, a $800 \mathrm{~ms}$ tone (70db SPL; CS) was coupled with a $80 \mathrm{~ms}$ airpuff administered to the cornea $(200 \mathrm{mmHg}$; US). The tone was presented first, with the airpuff beginning $720 \mathrm{~ms}$ after the tone so that both stimuli co-terminated (Figure 1b). In unreinforced trials, only the tone was presented. Inter-trial intervals fluctuated randomly between 10 and 14 seconds. The tone was presented through noise canceling headphones. The airpuff was delivered towards the eye's medial canthus through tubing affixed to a headband. During the delay eyeblink conditioning task, participants were instructed to fixate on a cross in the center of a computer screen and refrain from deliberate eye or body movement. Participants were informed that their attention would be measured at the experiment's conclusion, that they would hear some tones, and intermittently feel a puff of air in the eye that would not be painful or harmful. 


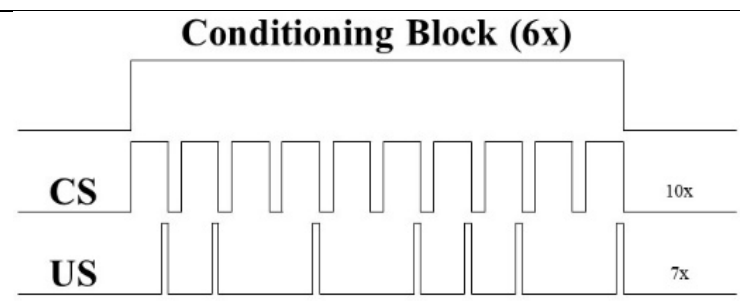

1a

\section{Conditioning Trial}

$800 \mathrm{~ms}$

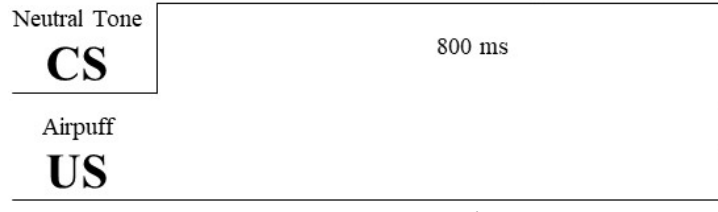

$1 \mathrm{~b}$

Figure 1. Visual representation of the experimental schedule depicting a conditioning block with $70 \%$ reinforcement schedule (5a) and timing of unconditioned stimulus (US) and conditioned stimulus (CS) presentation for an individual conditioning trial $(5 b)$.

Eyeblinks were recorded using an electrooculogram (EOG) amplifier (Gain=2000, 0.05 Hz) from a MP150 system (Biopac Systems, Inc.). Analysis of eyeblink data was performed offline with AcqKnowledge 5.0 (Biopac Systems Inc.). Using established conventions (Daum et al., 1993; Wolf et al., 2009), a blink was defined as a change in the EOG waveform's curve exceeding an amplitude of $25 \mathrm{~A} / \mathrm{D}$ units for $25 \mathrm{~ms}$ and a peak amplitude exceeding $75 \mathrm{~A} / \mathrm{D}$ units. A conditioned response was defined as a closing movement of the eyelid occurring between $450 \mathrm{~ms}-720 \mathrm{~ms}$ after tone onset. The first conditioned response and conditioned response frequency in each of the seven task blocks was determined for all participants.

With respect to the HRV analyses, R-R intervals were detected in the ECG data using automated routines in Acqknowledge 4.1 software (BIOPAC, Inc.). The R-wave file was then visually inspected to correct misidentified or omitted R-waves. Kubios 2.0 (Biosignal Analysis and Medical Imaging Group, University of Finland) was used to calculate beats-per-minute (BPM) and for spectral analysis of R-waves. R-R interval data were divided into two segments, one spanning the experimental manipulation period and the other spanning the eyeblink condition. HRV analyses were conducted on the entire segment length for each of these two conditions: a fast Fourier transform was applied separately to R-R interval data to extract normalized HF-HRV from a de-trended, end-tapered interbeat interval time series. The spectrum 
for the selected R-R interval segment was calculated via Welch's periodgram method, in which seconds (with a window overlap of 50\%). The HF-HRV ratio was selected as the primary focus for statistical analysis. Following Berntson (1997) and Malliani et al. (1994), we calculated HRV in normalized units to elucidate shifts in this frequency component that might otherwise be obscured by use of absolute units which are dependent on total HRV power. HF-HRV was averaged separately for the experimental manipulation and the eyeblink conditioning task periods.

\section{Results}

Repeated-measures ANOVA was used to examine the impact of Group (mindfulness vs. active listening) and Time (study sessions 1-6) on state mindfulness. A significant Group x Time interaction was observed $\left(\mathrm{F}_{5,235}=3.77, \mathrm{p}=.003, \eta^{2}=.07\right)$, indicating that mindfulness training increased state mindfulness across the six study sessions (Figure 2).

Figure 2. Mean state mindfulness ( \pm 1 standard error) over the course of six study sessions.
Participants in the mindfulness condition displayed higher state mindfulness in response to
experimental induction than participants in the active listening control condition. Delay
eyeblink conditioning took place during the sixth study session.


The mean trial number of first conditioned response, a measure of conditioning onset, is presented in Figure 3. Given that sex differences in eyeblink conditioning are well-documented (Wolf et al., 2009), a one-way ANCOVA was conducted to examine the impact of mindfulness training on first conditioned response, controlling for sex. There was a significant mean difference in the number of trails to first conditioned response $\left(\mathrm{F}_{1,46}=6.12, \mathrm{p}=.017, \eta^{2}=.12\right)$, indicating that mindfulness training delayed $(M=11.8, S D=19.57)$ conditioned response acquisition relative to the active listening $(M=2.5, S D=2.16)$.

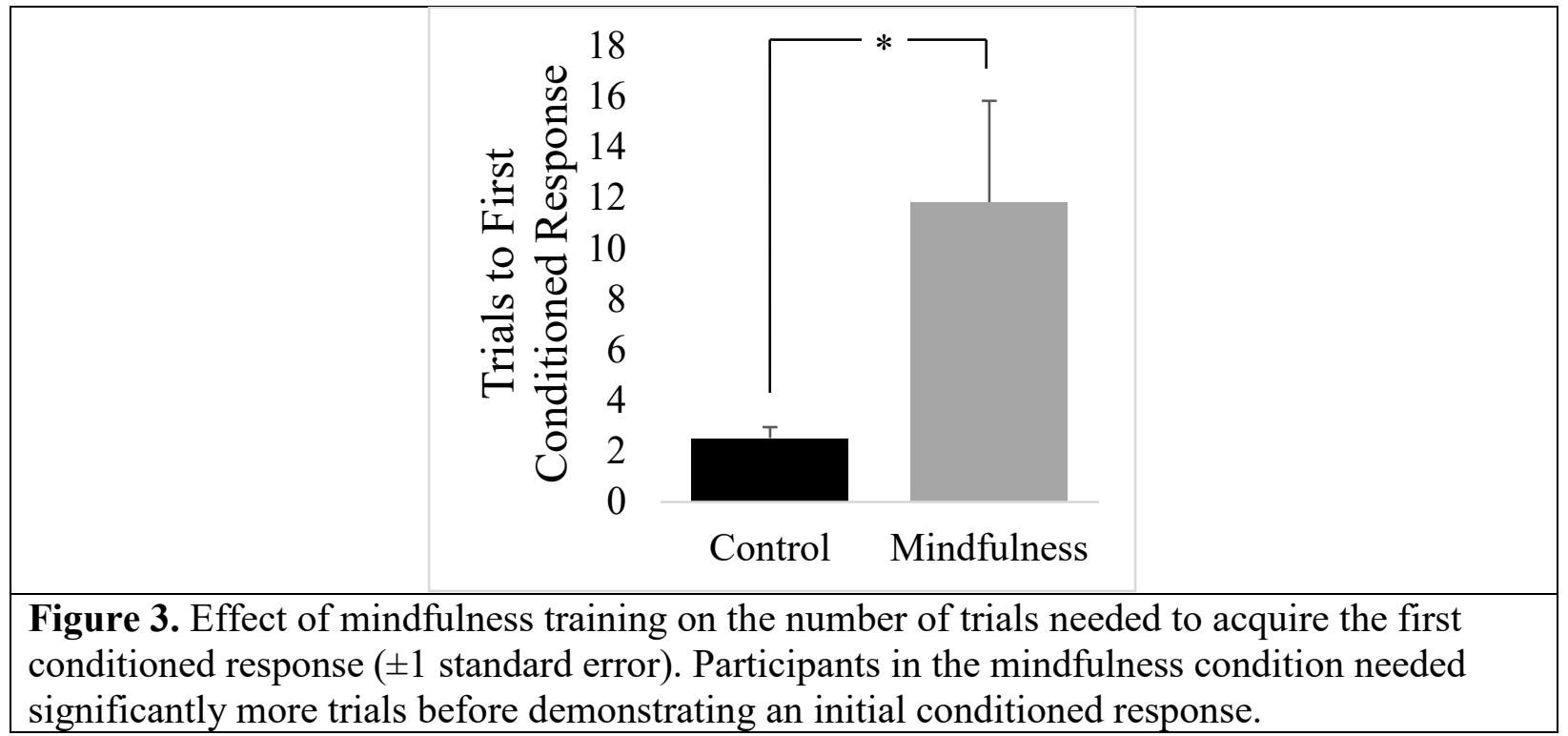

The development of conditioned response frequency across the six acquisition blocks and the extinction block is presented in Figure 4. For the acquisition blocks, repeated measures ANCOVA with factors Group (mindfulness versus active listening) and Block (blocks 1-6) yielded a significant main effect of Group, controlling for $\operatorname{sex}\left(\mathrm{F}_{1,46}=16.95, \mathrm{p}<.001, \eta^{2}=.27\right)$. Significantly higher conditioned response frequency was observed in the active listening condition compared with the mindfulness condition. 


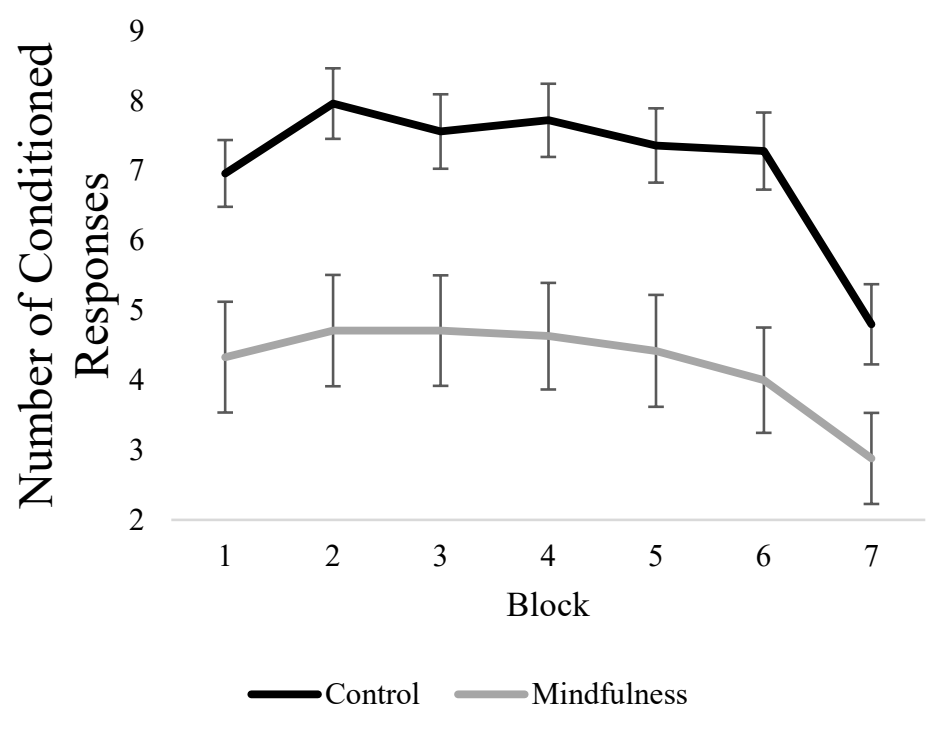

Figure 4. Effect of mindfulness training on acquisition and extinction of delay eyeblink conditioned responses ( \pm 1 standard error). Mindfulness training resulted in less conditioned responding.

Path analysis was used to examine whether training-dependent changes in state mindfulness explained reductions in conditioned responding (Figure 5). Experimental group was a significant predictor of change in state mindfulness from study session one to study session six, and change in state mindfulness was a significant predictor of conditioned response frequency.

CI: $0.48,6.69)$ on conditioned response frequency via change in state mindfulness. Because stress and autonomic regulation are known to influence delay eyeblink response (e.g., Wolf et al., 2009), a sensitivity analysis was performed to determine whether HF-HRV, a marker of vagal tone, could also serve as a mediator. A significant Group (mindfulness vs. active listening) X Condition (induction vs. eyeblink conditioning) interaction on HF-HRV was observed $\left(\mathrm{F}_{1,45}=6.062, \mathrm{p}=.018, \eta^{2}=.12\right)$, such that participants in the active listening condition experienced vagal withdrawal from induction to eyeblink conditioning whereas participants in the mindfulness condition experienced no such vagal withdrawal. In a sensitivity analysis conducted 
with a multivariate path model, change in state mindfulness remained a significant mediator

$200 \quad(B=4.34, S E=1.94,95 \%$ CI: 0.37, 7.14), whereas HF-HRV did not mediate the observed effect.

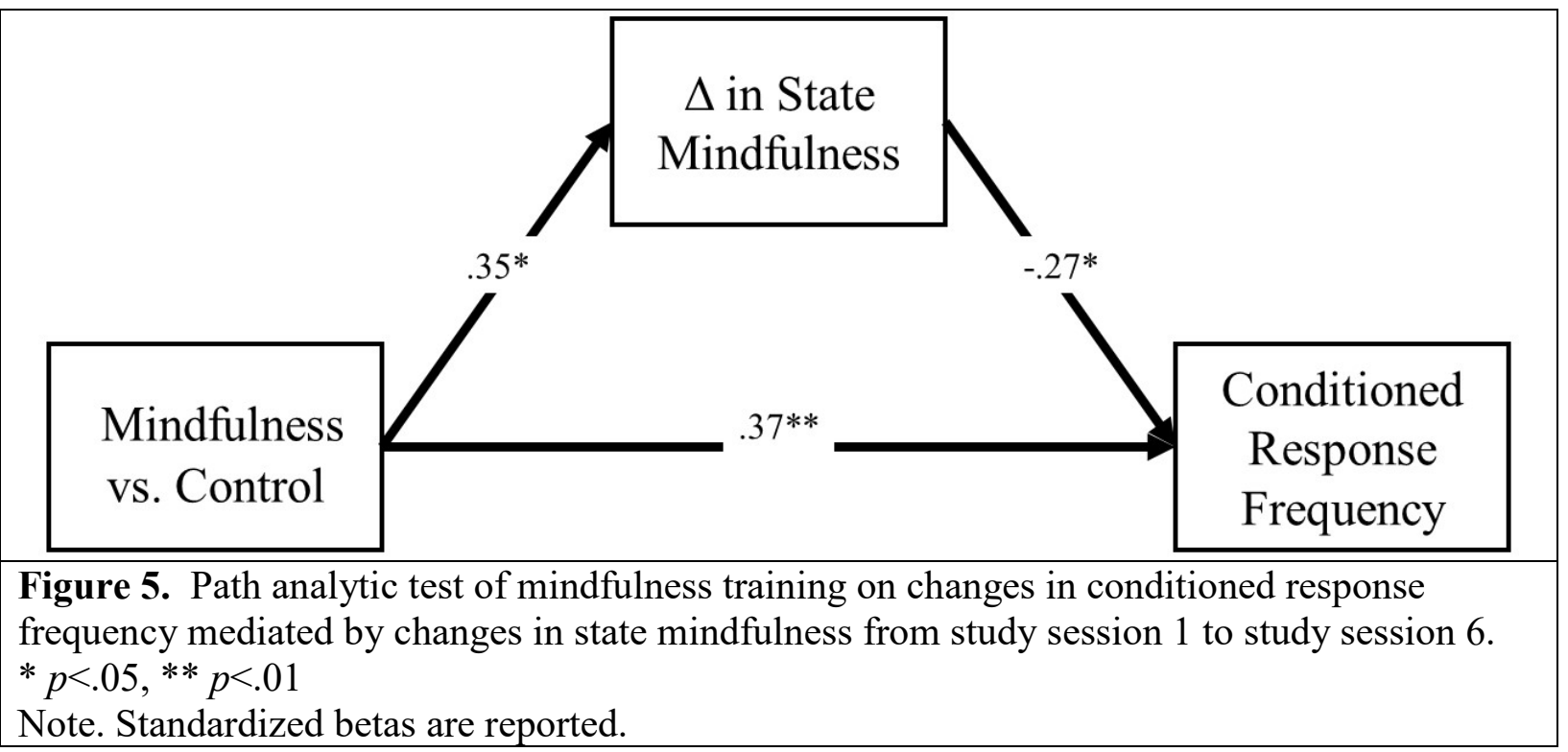

\section{Discussion}

This study assessed the effect of mindfulness training on Pavlovian (classical)

conditioning. Findings indicate that mindfulness decreased classically conditioned behavior.

Mindfulness training delayed the onset of first conditioned response (i.e., first blink) and decreased conditioned response frequency. To our knowledge, this is the first study to demonstrate effects of mindfulness training on the acquisition of conditioned responses. Furthermore, although mindfulness training buffered against vagal withdrawal induced during startle responses to the task, training-dependent increases in state mindfulness mediated the effect of mindfulness training on conditioned response frequency, even after accounting for changes in HRV at the time of conditioning. This mechanistic finding suggests that mindfulness training disrupts classical conditioning via the cognitive aspects of state mindfulness and not 
through mere parasympathetic regulation. However, neuroimaging is indicated to more fully elucidate the mechanisms by which mindfulness modulates conditioned responding.

Conditioned behaviors underlie some of the most vexing public health threats confronting society today. For instance, addiction is thought to arise in part through a process of classical conditioning in which drug-related contextual cues come to reflexively elicit drug use - a conditioned response that can operate out of habit and against one's will. Mindfulness training may be one method of increasing volitional control over habitual, automatized responding. Our results, in conjunction with strong theoretical support (Deikman, 1966; Garland et al., 2015; Hölzel et al., 2011) and associated empirical evidence (Kirk \& Montague, 2015; McHugh et al., 2012; Stillman et al., 2014; Whitmarsh et al., 2013), indicate that mindfulness meditation training disrupts conditioned behavior. Results from the present study further justify the use of mindfulness as a clinical intervention and may have far-reaching implications. In fact, findings from this study suggest that mindfulness training may have the potential to inoculate individuals against the development of maladaptive conditioned behaviors. In the general population, relaxing conditioned behavioral contingencies through mindfulness training may allow individuals to act with greater intentionality and purpose. In the context of psychopathology, pairing mindfulness training with established extinction therapy techniques might enhance treatment outcomes. However, continued examination is needed to better characterize how mindfulness meditation discourages the formation of maladaptive habits and how these findings can be more directly translated into clinical intervention approaches. 
237 Funding: Author was funded by a Varela Award from the Mind and Life Institute. Author was 238 funded by NIDA (R01DA042033) and NCCIH (R61AT009296). 


\section{References}

Berger, T. W., Alger, B., \& Thompson, R. F. (1976). Neuronal substrate of classical conditioning in the hippocampus. Science, 192(4238), 483-485.

Cheng, D. T., Disterhoft, J. F., Power, J. M., Ellis, D. A., \& Desmond, J. E. (2008). Neural substrates underlying human delay and trace eyeblink conditioning. Proceedings of the National Academy of Sciences, 105(23), 8108-8113.

Craske, M. G., Kircanski, K., Zelikowsky, M., Mystkowski, J., Chowdhury, N., \& Baker, A. (2008). Optimizing inhibitory learning during exposure therapy. Behaviour Research and Therapy, 46(1), 5-27. https://doi.org/10.1016/j.brat.2007.10.003

Daum, I., Schugens, M. M., Ackermann, H., Lutzenberger, W., Dichgans, J., \& Birbaumer, N. (1993). Classical conditioning after cerebellar lesions in humans. Behavioral Neuroscience, 107(5), 748.

Davey, G. C. (1992). Classical conditioning and the acquisition of human fears and phobias: A review and synthesis of the literature. Advances in Behaviour Research and Therapy, 14(1), 29-66.

Deikman, A. J. (1966). De-automatization and the mystic experience. Psychiatry, 29(4), 324338.

Garland, E. L., Baker, A. K., \& Howard, M. O. (2017). Mindfulness-oriented recovery enhancement reduces opioid attentional bias among prescription opioid-treated chronic pain patients. Journal of the Society for Social Work and Research, 8(4), 493-509.

Garland, E. L., Farb, N. A., R. Goldin, P., \& Fredrickson, B. L. (2015). Mindfulness broadens awareness and builds eudaimonic meaning: A process model of mindful positive emotion regulation. Psychological Inquiry, 26(4), 293-314. 
Hanh, T. N. (2009). Peaceful action, open heart: Lessons from the Lotus Sutra. Parallax Press.

Hofmann, S. G., \& Smits, J. A. (2008). Cognitive-behavioral therapy for adult anxiety disorders: a meta-analysis of randomized placebo-controlled trials. The Journal of Clinical Psychiatry, 69(4), 621.

Hogarth, L., Balleine, B. W., Corbit, L. H., \& Killcross, S. (2013). Associative learning mechanisms underpinning the transition from recreational drug use to addiction. Annals of the New York Academy of Sciences, 1282(1), 12-24.

Hölzel, B. K., Lazar, S. W., Gard, T., Schuman-Olivier, Z., Vago, D. R., \& Ott, U. (2011). How does mindfulness meditation work? Proposing mechanisms of action from a conceptual and neural perspective. Perspectives on Psychological Science, 6(6), 537-559.

Kabat-Zinn, J. (1990). Full Catastrophe Living. NY: Delacorte Press.

Kirk, U., \& Montague, P. R. (2015). Mindfulness meditation modulates reward prediction errors in a passive conditioning task. Frontiers in Psychology, 6, 90.

Lau, M. A., Bishop, S. R., Segal, Z. V., Buis, T., Anderson, N. D., Carlson, L., ... Devins, G. (2006). The Toronto mindfulness scale: Development and validation. Journal of Clinical Psychology, 62(12), 1445-1467.

Maren, S. (2011). Seeking a spotless mind: extinction, deconsolidation, and erasure of fear memory. Neuron, 70(5), 830-845. https://doi.org/10.1016/j.neuron.2011.04.023

McHugh, L., Procter, J., Herzog, M., Schock, A.-K., \& Reed, P. (2012). The effect of mindfulness on extinction and behavioral resurgence. Learning \& Behavior, 40(4), 405415.

Pavlov, P. I. (2010). Conditioned reflexes: an investigation of the physiological activity of the cerebral cortex. Annals of Neurosciences, 17(3), 136. 
Pitman, R. K. (1989). Post-traumatic stress disorder, hormones, and memory. Biological Psychiatry, 26(3), 221-223.

Powers, M. B., Halpern, J. M., Ferenschak, M. P., Gillihan, S. J., \& Foa, E. B. (2010). A metaanalytic review of prolonged exposure for posttraumatic stress disorder. Clinical Psychology Review, 30(6), 635-641.

Shapiro, S. L., Carlson, L. E., Astin, J. A., \& Freedman, B. (2006). Mechanisms of mindfulness. Journal of Clinical Psychology, 62(3), 373-386.

Stillman, C. M., Feldman, H., Wambach, C. G., Howard, J. H., \& Howard, D. V. (2014). Dispositional mindfulness is associated with reduced implicit learning. Consciousness and Cognition, 28, 141-150.

VanElzakker, M. B., Dahlgren, M. K., Davis, F. C., Dubois, S., \& Shin, L. M. (2014). From Pavlov to PTSD: the extinction of conditioned fear in rodents, humans, and anxiety disorders. Neurobiology of Learning and Memory, 113, 3-18.

Watson, J. B., \& Rayner, R. (1920). Conditioned emotional reactions. Journal of Experimental Psychology, 3(1), 1.

White, G. (2004). The illustrated natural history of Selborne. Thames \& Hudson.

Whitmarsh, S., Uddén, J., Barendregt, H., \& Petersson, K. M. (2013). Mindfulness reduces habitual responding based on implicit knowledge: Evidence from artificial grammar learning. Consciousness and Cognition, 22(3), 833-845.

Wikler, A. (1948). Recent progress in research on the neurophysiologic basis of morphine addiction. American Journal of Psychiatry, 105(5), 329-338. 
Wolf, O. T., Minnebusch, D., \& Daum, I. (2009). Stress impairs acquisition of delay eyeblink conditioning in men and women. Neurobiology of Learning and Memory, 91(4), 431436.

Zeidan, F., Johnson, S. K., Diamond, B. J., David, Z., \& Goolkasian, P. (2010). Mindfulness meditation improves cognition: Evidence of brief mental training. Consciousness and Cognition, 19(2), 597-605. 